\title{
Génies de brousse et Corybantes
}

ou comment introduire la diachronie dans la comparaison

\section{Renée Koch-Piettre}

\section{(2) OpenEdition}

\section{Journals}

Édition électronique

URL : http://journals.openedition.org/span/1700

DOI : $10.4000 /$ span. 1700

ISSN : 2268-1558

Éditeur

École pratique des hautes études. Sciences humaines

Édition imprimée

Date de publication : 1 janvier 2014

Pagination : $39-56$

ISBN : 9782909036434

ISSN : 0294-7080

Référence électronique

Renée Koch-Piettre, « Génies de brousse et Corybantes », Systèmes de pensée en Afrique noire [En ligne], 19 | 2014, mis en ligne le 06 février 2020, consulté le 10 février 2020. URL : http:// journals.openedition.org/span/1700 ; DOI : 10.4000/span.1700 


\section{Génies de brousse et Corybantes ou comment introduire la diachronie dans la comparaison}

\section{Renée Koch-Piettre}

Directrice d'études EPHE, Anthropologie et histoire du monde antique (ANHIMA)

Michel Cartry, ethnologue et philosophe, prenait le temps de tirer parti, au fur et à mesure, des déplacements de perspectives que les rencontres les plus inattendues, l'effort volontaire de changer soi-même de place et l'avancement de l'âge peuvent occasionner. "Pour l'instant, je n'y comprends rien ", tel aurait pu être son refrain. Relisons, de ce point de vue, le précieux article intitulé " D'un rite à l'autre : la mémoire du rituel et les remémorations de l'ethnologue ${ }^{1}$. Méditant une recommandation de Wittgenstein qui invitait à " tracer des lignes qui relient les composantes communes" des rites, et s'appuyant sur Pierre Smith et sa critique implicite de la doctrine lévi-straussienne selon laquelle le mythe s'oppose au rite comme les distinctions de la pensée à une tentative de retour à la continuité du vécu ${ }^{2}$, Michel Cartry repérait une relation, évidente pour les acteurs mais à ses propres yeux incompréhensible, entre un segment de rite funéraire gourmantché et ce même segment réapparaissant d'une part dans le rituel initiatique des jeunes garçons et d'autre part dans les rites de naissance. Ce qui se passe alors peut au moins, suggérait-il, être comparé à ce moment où, dans la composition d'une fugue musicale, des voix qui se répondent "entrent en imitation".

Claude Lévi-Strauss avait réservé au système synchronique du mythe la métaphore musicale. Michel Cartry montre que le rite lui aussi se pense lui-même, comme une

${ }^{1}$ Cartry, 2006.

2 Lévi.Strauss, 1971 : 603. 
${ }^{3}$ Smith. 1979 : p. 141 168.

${ }^{4}$ On peut songer aussi à l'accueil de l'initié à son retour au village et dans sa famille, comme pour une renaissance. composition musicale, mais il se pense en diachronie, dans la durée longue du destin individuel et de la vie des sociétés. Le rite n'est pas seulement une "boîte noire ", un "piège à pensée ", selon le mot de Pierre Smith ${ }^{3}$. C'est ce qu'il lui a été donné de comprendre, au sens le plus fort de ce terme, d'éprouver sans doute comme une initiation qui déplace entièrement les perspectives dont on se satisfaisait jusque-là, le jour où il reconnut, lors d'un chant de deuil entonné par les femmes dans la cour du mort et dansé, mimé par la fille aînée du mort, des formules caractéristiques d'un segment de l'initiation masculine, dont l'accès est pourtant formellement interdit aux femmes. Avec notamment cette réponse à une question sur le secret de l'enclos initiatique :

"ll n'y a rien dans l'enclos où nous sommes assis, sauf le cri de joie des femmes ".

Le mime de la fille aînée du mort, qui imite les gestes de l'initiation masculine, provoque en outre les rires de l'assistance féminine. Un ethnologue moins consciencieux aurait immédiatement repéré une inversion des rôles masculin et féminin caractéristique des rites d'initiation selon Arnold Van Gennep et s'en serait contenté. Michel Cartry ne s'arrête pas là, il repère plutôt cette indétermination de l'identité, cette "suspension des différences " marquant ces moments de la vie où l'on perd brutalement le confort acquis de son statut antérieur : l'initié arraché au cocon de l'enfance et mis face à la trivialité des objets de l'initiation jusque-là entourés de tant de mystère et de prestige, l'enfant des deux sexes devenu orphelin et projeté dans un rôle nouveau, éprouvent sur ce plan un même abandon, " une même sensation de froid ". Une même "expérience de la perte ". Michel Cartry ne va pas jusqu'à comparer cette expérience au vécu du nouveau-né dont l'image me semble pourtant appelée par la mention du "cri de joie des femmes " ${ }^{4}$ l'ethnologue se place en retrait et laisse au lecteur, ou peut-être à une reprise ultérieure de ce texte qui ne trouva pas le temps de sa mise en ceuvre, le soin de déployer à ses risques et périls les connexions que le matériel ethnographique appelait, peut-être, de lui-même - mais il faut toujours se méfier des évidences. 
La recherche comparative que j'ai partagée durant une dizaine d'années avec M. Cartry et un petit groupe de fidèles concernait ce que nous appelions la " mise en présence » des instances sur l'aire sacrificielle. Nos cheminements rituels nous ont permis de tracer une ligne, en l'occurrence, entre la ligature, l'écriture et le sacrifice. Soulignons l'énorme déplacement de perspectives qui est exigé d'un helléniste aventuré du côté du sacrifice en Afrique noire, une matière que Michel Cartry a très amplement explorée par ailleurs dans un ouvrage collectif de ce nom. En voici, par exemple, une illustration en forme de chimère, que je construis à partir d'informations ethnographiques disparates recueillies auprès de Michel Cartry au sujet de l'aire gourmantché au Burkina Faso : trois brins d'herbe noués ensemble, cela peut être appelé un autel, quand ce serait sur ce nœud, à l'emplacement peut-être d'un carrefour trifide où se rencontrent trois sentiers, que le sacrifiant peut susciter/rencontrer l'instance surnaturelle; le sacrifiant opérera cette rencontre par ses propres paroles de salutation et de prière et par le simple geste de verser là-dessus la matière oblatoire, et notamment le sang d'un animal; mais il pourra même y verser la substance d'un œuf, un " poulet " virtuel dont il énoncera les improbables caractéristiques exigées par l'oracle du devin, et inscrites, lorsqu'il s'agit d'un "sacrifice d'attache ", avec la spécification de l'instance destinataire, sur la face concave d'un morceau de calebasse concentrant les fonctions d'aide-mémoire, de fragment d'univers et d'objet destinal.

Au titre de mon " terrain " d'helléniste et pour suivre ces réflexions introductives sur la structure fuguée du rite, j'emprunte à mon tour ici mon objet au champ de l'initiation: la comparaison entre initiations de jeunes gens en Afrique noire et traces de rites initiatiques en Grèce ancienne a fait l'objet d'un gros ouvrage de l'historien des religions Henri Jeanmaire, publié en 1939 sous le titre Couroi et Courètes ${ }^{5}$. L'.historien y interrogeait avec soin la littérature ethnographique de son temps sur l'Afrique noire, repérant une opposition entre initiations systématiques de classes d'âges et initiations " mystériques " de confréries : les- ${ }^{5}$ Jeanmaire, 1939. 
${ }^{6}$ Et ce ne serait encore qu'un moindre mal, s'il ne fallait y ajouter encore les noms des Cabires, des Telchines, des Dactyles et des Kyrbantes...

${ }^{7}$ Strabon, X, 3, 1.23. quelles peuvent cependant, comme dans le komo bambara, intéresser à la longue tous les adultes d'une société et finir par se confondre avec l'initiation tribale. La comparaison rigoureusement conduite jetait une lumière nouvelle sur la périlleuse question grecque des "Courètes " : s'appuyant sur l'étymologie la plus obvie qui reconnaît dans ce mot le nom des jeunes garçons, ko(u)roi, ainsi dénommés parce qu'on leur rasait partiellement les cheveux (koura) dans un âge qui correspondait à celui des rites d'adolescence, Jeanmaire conduisait son analyse des Courètes d'Homère à la geste de Thésée, aux initiations crétoises et à l'éducation spartiate. Ce faisant, en se concentrant sur les kouroi, il évitait aussi un piège qui n'a cessé de barrer aux hellénistes l'accès à la question : la recherche des correspondances entre Courètes et Corybantes, deux noms que les Grecs ont mêlés et souvent confondus ${ }^{6}$ tout en s'efforçant de leur assigner des localisations géographiques ou des environnements mythiques ou cultuels distincts. Outre une vague paronomase, ce qui a conduit la tradition à cette confusion, c'est qu'il s'agissait dans les deux cas d'un groupe de daimones aux danses bruyantes et tumultueuses, dont le théâtre mythique se situe sur un certain mont Ida; les Courètes, serviteurs de Rhéa, mère de Zeus, couvraient ainsi les vagissements de son bébé, sur l'Ida de Crète; les Corybantes, serviteurs de Cybèle ou de la Mère des Dieux, accompagnaient de leur frénésie l'épiphanie de la déesse sur l'Ida troyen ou phrygien. Un long exposé " théologique " du géographe ancien Strabon à l'occasion d'un excursus dans sa description de la Crète ${ }^{7}$ achève de brouiller le paysage pour les chercheurs. Il pouvait apparaître cependant que Strabon privilégiait, dans son enquête, le discours de la mythologie, et que celle-ci l'orientait vers ce que Jeanmaire aurait pu appeler une confrérie mystérique, au lieu de l'initiation adolescente : une confrérie d'initiés incarnant une troupe de démons ou dieux et requérant euxmêmes un culte orgiastique, ou bien le cortège enthousiaste des desservants et adorateurs d'une divinité.

L'initiation ouvre un champ de rencontre des instances surnaturelles que je m'aventure à définir comme opposé et complémentaire de celui du sacrifice : en ceci 
que dans le cas du sacrifice combiné avec la divination, le " chemin du rite " est bordé, balisé d'avance ou précisément défini à mesure pour éviter autant que possible tout accident, mais qu'il est abordé comme un territoire inconnu et semé d'imprévu dans le cas de l'initiation. Il en va ainsi du vécu ordinaire de l'initiant adolescent, mais plus encore de l'homme qu'un accident de la vie contraint de recevoir une initiation spécifique qui l'attachera au culte du génie perturbateur. Dans l'espace gourmantché étudié par Michel Cartry, l'homme, à travers certaines des composantes multiples de sa personne, peut en effet à l'improviste entrer en relation avec l'invisible sous l'espèce des morts, des ancêtres, et de lieux marqués dans l'espace naturel, comme une colline, un arbre, un rocher, une rivière, une source, habités par ce que la langue française nommerait génie ou esprit de brousse. Leur rencontre ou malencontre se reconnaît à des dérangements de tous ordres qui imposent à leur victime involontaire un traitement rituel à la fois cathartique et initiatique, et la rivent au culte initié par ce traitement (en Grèce, on parlerait, en des circonstances voisines, de nympholepsie, de panolepsie, de mètrolepsie, d'épilepsie... ou encore de quelque démon vengeur comme les alastores ou elastores, mais chacun de ces termes relève de contextes précis et il convient de ne pas tout confondre $)^{8}$. L'initiation des jeunes garçons, qu'étudie Jeanmaire, provoque délibérément et systématiquement un tel contact avec les instances surnaturelles. Ce contact est tel que, notait Jeanmaire, les initiants "sont pour un temps, eux-mêmes, ces puissances et ces esprits incarnés " sous le masque, l'enduit luisant, les vêtements de fibres et de feuillages, avec leurs gesticulations impressionnantes au son des trompes et dans le grondement tournoyant des rhombes.

Accaparé par la comparaison qu'il opère avec les rites africains d'initiation adolescente, et focalisé sur la seule dénomination de "Courètes ", si proche de Kouroi, les jeunes gens, Jeanmaire laissait ouverte la question des Corybantes, qu'il nous semble pouvoir situer à mi-chemin du traitement rituel d'une malencontre et de mystères qui agrègent l'impétrant à une confrérie initiatique. Nous orientons, quant à nous, notre observation vers ces Corybantes qui nous font notam-

8 Précisions simple. ment qu'il s'agit d'attaques du dieu Pan, de la Mère des dieux, des Nymphes, ou de quelque démon suscité par une faute ou souillure anciennes et persécutant le fautif ou sa descendance. 
${ }^{9}$ Par ex. dans les

Nuées, v. 254sq., ou les Guêpes, v. 119sq.

10 Voir son Hélène (datée de 412); ses Crétois consacrés à la légende du Minotaure sont largement antérieurs (435).

11 Voutiras, 1996 243.256 (Macédoine).

12 Voir la récapitu. lation de Ustinova, 1992.1998 : 503.520. Également Blakely. $2000: 119.127$.

13 Linforth,

$1946: 121 \cdot 162$,

qu'analysa Festugière, 1946 : 493.495 et que reprend brièvement Dodds, 1977 : 84.86, avec notes

${ }^{14}$ Les traductions sont adaptées par nous de la collection « des

Universités de France (Les Belles Lettres), sauf celles de l'lon (trad. Robin dans la collection " Pléiade "), et celle du Minos (nous traduisons). ment entrer dans les émotions de nombreux Athéniens aux alentours de la fin du $v^{e}$ siècle av. notre ère, au moment d'un tournant de l'histoire proprement destinal. Et nous choisissons de nous concentrer sur quelques passages de Platon.

Il semble que, du vivant de Socrate, l'initiation aux mystères des Corybantes ait constitué une sorte de mode dont témoignent encore Aristophane ${ }^{9}$ ou l'une des dernières pièces d'Euripide ${ }^{10}$. Les mystères avaient vraisemblablement un caractère domestique et privé ${ }^{11}$, même s'il a existé en Asie Mineure, des prêtrises publiques (payantes) des Corybantes ${ }^{12}$. La question de leur occurrence chez Platon a été traitée de manière approfondie par Ivan Mortimer Linforth il y a une soixantaine d'années ${ }^{13}$, mais il ne nous semble pas inutile d'en reprendre le dossier.

Le plus connu des passages de Platon sur les Corybantes se situe à la fin du Criton. En une prosopopée célèbre, Socrate vient d'imaginer que les Lois d'Athènes s'adressent à lui, lui exposent sa dette à leur égard et le convainquent de ne pas se dérober par la fuite à la sentence de mort prononcée contre lui. "Voilà, conclut-il, ce que moi, je crois entendre, comme ceux qui sont possédés par les Corybantes (hoi korubantiôntes) croient entendre des flûtes (aulôn); oui, le son de ces paroles bourdonne (bombei) en moi et m'empêche de rien entendre d'autre ${ }^{14}$."

Les korubantiôntes dont il est question, ce sont ceux qui "font les Corybantes " ou "sont possédés par les Corybantes " ou "subissent le traitement corybantique ». On rencontre un verbe korubantizein (Aristophane, Guêpes, 119) pour dire l'acte de provoquer cette possession, d'infliger ce traitement. De manière répétée, la possession corybantique est d'autre part corrélée à la musique d'auloi, ces vents à anche et à deux tuyaux, au son strident ou bourdonnant (bombei), associés notamment à la transe bachique, dionysiaque.

Que les arguments, les raisonnements très sensés, les logoi des Lois puissent aux yeux de Socrate ressembler aux stridences et au bourdonnement d'une musique extatique, 
voilà qui a de quoi étonner ${ }^{15}$. Nous sommes moins surpris de rencontrer la même image quand il s'agit de sophistes faisant, de leurs pirouettes verbales, tourner la tête à un naïf jeune homme. Ainsi dans l'Euthydème, Socrate rassure le jeune Clinias en ramenant de l'inconnu (ici l'argumentation sophistique qui terrifie le jeune homme) à du connu (le rappel des préliminaires de son expérience initiatique, qui l'effrayaient autrefois mais n'étaient que jeu, et dont la description ici se précise) :

"Peut-être ne vois-tu pas ce que les deux étrangers sont en train de faire autour de toi. Ils font exactement comme dans l'initiation des Corybantes (hoi en têi teletêi tôn Korubantôn), quand on organise la cérémonie de l'intronisation (tèn thronôsin) autour du futur initié. On procède alors à des danses et à des jeux (choreia tis esti kai paidia), comme tu dois le savoir si tu as reçu l'initiation (ei ara kai tetelesai). En ce moment ces deux hommes ne font que danser (choreueton) autour de toi, et comme jouer en menant une ronde (hoion orcheisthon paizonte), pour t'initier ensuite "(277de).

La conditionnelle en incise, " comme tu dois le savoir si tu as reçu l'initiation (ei ara kai tetelesai) ", montre que, si l'initiation corybantique est chose répandue et supposée connue du jeune Clinias, elle n'était pas pour autant systématique, mais appliquée aux seuls malades ${ }^{16}$ supposés en avoir besoin. Ce moment qui fait tourner la tête dans l'initiation corybantique, ce serait donc celui qui précède l'initiation proprement dite, à laquelle c'est alors Socrate lui-même qui va procéder au bénéfice du jeune Clinias, feignant de croire que jusque-là les deux sophistes n'avaient encore fait que jouer, et expliquant que le même mot utilisé de manière sibylline par les deux sophistes, manthanein, peut signifier aussi bien apprendre (ce qu'on ne savait pas) que comprendre (sunienai), ce qu'on savait déjà.

Le moment du rituel initiatique préliminaire ainsi évoqué est nommé thronôsis, intronisation : le futur initié était placé sur un siège, un trône, et on lui faisait tourner la tête par les danses virevoltantes et autres jeux. L'initiation elle-même est chose sérieuse et vient ensuite. Et Socrate insiste :

\footnotetext{
${ }^{15}$ Voir cependant déjà le Charmide, où un Socrate rentrant des combats de 432 autour de Potidée témoigne dans un gymnase athénien des logoi que lui ont appris, en guise de médecine, les incantations (epôidai) d'un Thrace instruit par le « roi » Zalmoxis.

${ }^{16}$ Nosos, la maiadie, nosein, ètre malade, sont des mots couramment associés à l'initiation coryban. tique : voir par ex. Aristophane, Guêpes 114 . Nous y revenons infra.
} 
[à s'en tenir à ces préliminaires], " on serait seulement en état de badiner avec les gens, en utilisant les divers sens des mots pour leur donner des crocs-en-jambe et les renverser, comme ceux qui s'amusent à vous retirer les tabourets au moment où vous allez vous asseoir, puis rient de vous voir culbuter à la renverse."

Cependant, il apparaît que, même dans la partie sérieuse de l'initiation elle-même, l'initiant reste sous l'emprise de la fièvre qui l'a saisi lors des préliminaires, à ceci près que désormais il est littéralement possédé lui-même, il est entré lui-même dans la danse des corybantes. C'est ce que laisse entendre cette comparaison par exemple de Platon :

" De mème que ceux qui sont possédés par les Corybantes ne se livrent pas à leurs danses quand ils ont tous leurs esprits, de même aussi les auteurs de chants lyriques n'ont pas tous leurs esprits quand ils composent leurs chants magnificues " Platon, lon, 533 e-534a.

Comment passe-t-on des préliminaires de la thronôsis à la possession elle-même? Y a-t-il évolution systématique de l'étourdissement provoqué à la possession? Divers textes laissent entendre deux choses : d'une part, l'initiation corybantique et le délire qui l'accompagnent constituent le traitement d'une maladie, une sorte de purgation, de katharsis. D'autre part, le traitement en question est de nature homéopathique, je veux dire qu'on traite le mal par le mal, après identification de sa source. Prenons ce passage de Platon :

"S'il lsc. Phedre] s'en allait hors les murs, c'était pour s'exercer à le |sc. le discours de Lysias| réviter! Or, voici yu'il tombe sur un homme dont c'est la maladie d'écouter des discours (tôi nosounti peri logơon akoên); en le voyant, il s'est réjoui d'avoir là celui qui s'associerait à son délire corybantique (ton sugkorubantiônta) [...] celui qui se passionne pour les discours (tôn logôn erastou). „Platon, Phèdre, 228bc.

Le participe nosounti, "celui qui est malade ", ne laisse aucun doute sur la vertu thérapeutique de l'initiation. Mais s'y ajoute le diagnostic, qui spécifie la pathologie et la lie à une forme d'enthousiasme, ici l'amour (l'erôs, contenu dans le mot erastês, l'amoureux, l'amant). Phèdre rencontre, en Socrate, un malade atteint de la même pathologie que la sienne. Sans surprise pour qui se souvient du Criton, cette maladie consiste à être possédé par l'amour des discours, 
des logoi. Et comment la soigne-t-on? En berçant le malade de cela même qui cause son mal, en faisant coïncider la cause intérieure avec une cause extérieure, comme on berce vigoureusement le berceau du bébé agité, et donc, ici, en soignant l'amour du discours par les discours eux-mêmes dont s'enchantera encore le Socrate du Criton :

« [Les tout petits] devraient, s'il se pouvait, vivre sans cesse bercés comme dans un navire; voilà, pour les nourrissons nouveau-nés, l'idéal qu'il faut essayer de réaliser au plus près. Certains indices nous obligent d'ailleurs à conjecturer que l'expérience a révélé cette méthode et fait connaître ses avantages aux nourrices des petits aussi bien qu'à celles qui initient dans le cas des traitements corybantiques (hai peri ta tôn Korubantôn iamata telousai). Quand les mères, en effet, veulent endormir des enfants qui ont le sommeil difficile, au lieu de repos, c'est, au contraire, du mouvement qu'elles leur donnent en les secouant sans cesse dans leurs bras, et, au lieu du silence, un chantonnement : disons que, au plein sens du mot, elles enchantent comme par le son des flûtes (kataulousi) leurs bébés comme on enchanterait les bacchants hors de sens (tôn ekphronón bakcheiôn), en les traitant par le mouvement combiné de la danse et de la musique. "Lois (VII, 790 d-e).

Un peu plus loin, vient l'explication :

"Le mal des uns comme des autres est, en somme, de la peur, une peur qui vient d'une certaine mauvaise attitude de l'âme. Quand donc on oppose à de telles agitations une secousse extérieure, le mouvement qui vient du dehors maitrise le mouvement interne de frayeur et de folie, et, le maîtrisant, se trouve avoir ramené dans l'âme le calme et la tranquillité que troublaient, chez les uns comme chez les autres, les bonds pénibles du cœur. Or, c'est là un grand bienfait. Il procure, aux uns, le sommeil; il réveille les autres par les rondes et les flûtes et, avec le secours des dieux (orchoumenous te kai aulomenous meta theôn) auxquels chacun d'eux offre des sacrifices de propitiation, les ramène de la folie au bon sens " (anti manikôn hêmin diatheseôn hexeis emphronas echein). (790e-791b).

Remarquons au passage le trait d'union qui pourrait être fait ici entre les Courètes dansant autour du bébé Zeus, et les Corybantes virevoltant autour de l'initiant. L'agitation intérieure qui cause le mal est soignée par une agitation extérieure de même nature, qui, créant une forme d'homéostasie, produit un semblant de calme au milieu de la tempête, comme dans l'œil d'un cyclone, et rassoit les esprits, leur rend, pour le moins, des attitudes (hexeis) de bon sens. Même 
${ }^{17}$ Par exemple Detienne 2007 [1989].

${ }^{18}$ Reste que sur le territoire athénien l'ini. tiation corybantique ne paraît pas explicitement associée à la Mère des dieux : voir par ex. Ustinova, 1992.1998 510, n. 39.

${ }^{19}$ Question débattue mais importante : voir par ex. Thompson \& Wycherley, 1972, p. 25.38; Miller, 1995 : 131.156: Shear, Jr, 1995 :157.190; Borgeaud, 1996: Blomart, 2000-2001 : 9.22; Sickinger, 2004 : 93.109. si, commenterons-nous, le bouleversement où l'on se trouve pris a abouti à ce que l'on se tienne désormais cul par-dessus tête, à prendre la vie pour la mort et la mort pour la vie, par exemple, comme on l'entend dire dès Pindare et dans les premières traces de l'orphisme ${ }^{17}$.

Mais Platon nous apprend encore quelque chose de plus, c'est qu'au principe de l'agitation il y a une peur. Peur de quoi? Rappelons simplement qu'au temps de l'invasion de cette frénésie corybantique en Attique, une invasion qu'illustrerait un passage de l'Hélène d'Euripide, datée de 412, où l'on voit déferler sur les sommets de l'Ida le cortège bruyant de la Mère des dieux (1301-1368) ${ }^{18}$, Athènes venait de connaître le double traumatisme du désastre de Sicile et d'une parenthèse oligarchique violente. Ses longs murs qui lui faisaient une route sécurisée vers son port avaient été rasés. Le ressaisissement avait consisté, dans les dernières années du siècle, à restaurer la démocratie sur le fondement de ses lois les plus anciennes et les plus incontestées, celles de Dracon et de Solon, augmentées de toutes celles sur lesquelles un accord avait pu être démocratiquement obtenu. Athènes se constituait, dans le même temps, un lieu unique et centralisé pour ses archives publiques, à savoir précisément le Mètrôion, le sanctuaire de la Mère des dieux établi dans les murs mêmes de l'ancien Bouleutêrion, le lieu de réunion du Conseil de la cité simultanément installé dans un nouveau bâtiment juste à côté ${ }^{19}$. L'invasion de la Mère et de ses desservants les Corybantes coïncidait donc avec une centralisation et une uniformisation de la Loi athénienne, la cité avait trouvé l'apaisement dans le mal lui-même. On s'étonne moins, dans ces conditions, que Socrate ait pu se dire possédé comme dans l'initiation corybantique par les discours des Lois d'Athènes. Au niveau individuel, la centralisation des textes se traduisait par une intériorisation de la loi vécue, au moins par Socrate, comme une possession.

Ici, il nous faut encore tirer de Platon une information supplémentaire. L'homéostasie dont nous parlions n'était obtenue, nous semblait-il, qu'après que la cause du mal - une peur, donc - avait pu être identifiée et diagnostiquée. Mais pas seulement : ce diagnostic s'opérait au moyen de 
la possession elle-même, celle-ci n'intervenant, pour chaque initiant, que dans la mesure où celui-ci s'était montré réceptif à une musique particulière, un air de flûte parmi d'autres, qui simultanément révélait sa nature particulière et le genre de remède qui lui était propre. Ainsi, le rhapsode Ion ne réagit qu'aux vers d'Homère, n'est possédé qu'à leur écoute, comme les Corybantes ne sont possédés que par le dieu auquel ils se découvrent particulièrement rattachés (Platon, Ion, 536c) :

"À la façon de ceux qu'agite le délire Corybantique et qui ne perçoivent avec acuité qu'un seul air, quel qu'il soit, du Dieu auquel il leur échoit d'être possédés, et qui n'ont pas de peine à trouver des attitudes et des paroles allant à cet air, tandis que des autres ils n'ont cure, toi aussi, Ion, quand il t'arrive d'entendre qu'on parle d'Homère, tu es de même plein de ressources, et sans ressources au contraire si c'est des autres!"

Aristote confirme la vertu thérapeutique des chants pour les seules natures réceptives (Politique VIII, 4) : il vient de distinguer des mélodies éthiques, pratiques et enthousiastes, alors que plus haut il rapportait le mythe de l'invention de l'aulos par Athéna, et poursuit (1342a) :

"Les mouvements que ressentent avec force certaines âmes se retrouvent en toutes avec moins ou plus d'intensité - ainsi la pitié et la crainte, ou encore l'enthousiasme " -, car certains individus sont plus facilement possédés par cette sorte de mouvement, et nous voyons ces gens-là, sous l'effet des chants sacrés, quand ils ont recours à ces chants qui mettent l'âme hors d'elle-même, recouvrer leur calme comme sous l'action d'un traitement médical ou d'une purification" $=$

(kai gar hupo tautês tês kinêseôs katakôchimoi tines eisin, ek tôn d'hierôn melôn horômen toutous, hotan chrêsôntai tois exorgiazousi tên psuchên melesin, kathistamenous hôsper iatreias tuchontas kai katharseôs).

Cette réceptivité n'est pas encore, en soi, le traitement du mal : elle ne fait que révéler, nous disent deux autres passages, "ceux qui ont besoin de l'initiation " (il y a donc des gens qui ne se montrent nullement réceptifs) : dans le Pseudo-Platon, Minos 318b, il est question du bon législateur dans l'art de la flûte, il s'agirait de Marsyas:

" et son bien-aimé Olympos le Phrygien .[...] Leurs mélodies de flûte (aulémata) sont véritablement divines; seules, elles mettent en mouvement (kinei) et révèlent (ekphainei) ceux qui ont besoin de l'assistance des dieux (tous tôn theôn en chreiai ontas) ". 
On notera au passage la mention de l'origine phrygienne du flûtiste Olympos : c'est de Phrygie aussi que vient la Mère des dieux avec son cortège de Corybantes. La citation reprend presque mot pour mot ce passage du Banquet, 214c-e (c'est Alcibiade qui parle) :

"Ce que jouait Olympos, je dis moi que c'était de Marsyas, qui fut son maître. Et ses airs, qu'ils soient joués par un bon artiste ou par une pauvre joueuse de flûte, sont seuls capables de nous posséder (katechesthai), et de révéler ceux qui ont besoin des dieux et des initiations, car ces airs sont divins " (kai dêloi tous tôn theôn te kai teletôn deomenous).

Récapitulons : à des airs de flûte spécifiques venus de Phrygie, au moment, peut-être, de la thronôsis, les malades qui manifestent leur réceptivité par une transe également spécifique font voir par là même qu'ils ont besoin, en guise de thérapeutique, de l'initiation spécifique, qui consiste précisément dans le recours à ces mêmes danses et airs de flûte, associés à des divinités spécifiques (orchoumenous te kai aulomenous meta theôn) auxquelles chacun d'eux individuellement (hekastoi) offre finalement (ou simultanément), selon qu'il relève d'une divinité ou d'une autre, des sacrifices de propitiation (hois an kallierountes hekastoi thuôsi, voir le passage de Lois VII, 791a).

C'est sans doute sur cette base et selon cet exemple rituel à la mode de son temps que Platon, dans l'Ion ou dans le Phèdre, associe à des catégories d'âmes particulières telle forme d'enthousiasme, c'est-à-dire de délire possessionnel, plutôt que d'autres, ajoutant cette explication que, pour ce qui est du délire d'amour, il se porte vers des jeunes gens dont les âmes sont elles-mêmes parentes de la leur, en ce qu'elles ont contemplé et suivi en cortège, dans une vie antérieure, tel dieu dont ils étaient eux-mêmes un choreute et un desservant ${ }^{20}$. Nous avons là une interprétation proprement platonicienne de ce que l'initiation corybantique traitait par le diagnostic possessionnel et par des sacrifices appropriés à la divinité qui s'était révélée comme le destinataire compétent. Mais Platon affirme en outre qu'un tel

${ }^{20}$ Platon, Phèdre, 252c 253c. lien, réveillé, comme par réminiscence, par l'air de flûte approprié, a quelque chose d'intangible, de divin, d'éternel 
et de nécessaire, inaccessible aux variations du talent des flûtistes, aux aléas de la performance :

"Ce que jouait Olympos, je dis moi que c'était de Marsyas, qui fut son maître. Et ses airs, qu'ils soient joués par un bon artiste ou par une pauvre joueuse de flute, sont seuls capables de nous saisir profondément (katechesthai), et de révéler ceux qui ont besoin des dieux et des initiations, car ces airs sont divins " (kai dêloi tous tôn theôn te kai reletôn deomenous). Platon, Banquet, 214c-e (Alcibiade)

Cette précision gomme l'efficace relevant de la performance, de l'exécution du rite, pour attacher chacun à une vérité qui lui est définitivement propre et à laquelle il ne lui sera plus possible d'échapper, qu'il retrouvera partout aussi bien en lui-même qu'au-dehors. C'est ce qui arrive à Alcibiade quand il se découvre amoureux de Socrate, pris de frénésie corybantique à l'écoute des discours de Socrate, et de ceux-là seuls, de quelque trivialité qu'ils s'habillent; le passage a l'avantage, en outre, de préciser la montée de l'émotion dans la transe (Banquet, 214d-e : [Alcibiade]) :

\footnotetext{
"Si je ne devais pas vous sembler tout il fait ivre, je vous aurais dit - sous la foi du serment - quelle impression j'ai ressentie, et je ressens maintenant encore, à entendre ses discours. Quand je l'écoute, en effet, mon coeur hondit (pêdaii) plus fort que celui des possédés des Corybantes (tôn korubantióntón), ses paroles font couler mes larmes, et bien des gens, je le vois, éprouvent les mêmes impressions, "
}

Impression que ne lui font ni les paroles de Périclès ni celles d'aucun autre, mais celles de Socrate uniquement. Alcibiade a beau chercher à fuir, il a trouvé son lieu et son maître, il y revient toujours :

"Je me fais donc violence, je me bouche les oreilles comme pour échapper aux Sirènes, je m'èloigne, je fuis, pour éviter do rester là, assis près de lui, jusqu'à mes vieux jours... Souvent j'aurais plaisir à ne plus le voir en ce monde, mais si cela arrivait je sais que je serais encore plus malheureux " (Banquet, 216a)

Rappelons le mouvement général du dialogue du Banquet : Socrate s'y donne d'abord pour l'amoureux, pour le possédé, pourrait-on dire, de frénésie d'amour, analogue au délire corybantique, jusqu'au moment où Alcibiade révèle qu'en réalité ce sont ses auditeurs qui sont possédés par Socrate, amoureux de lui, que c'est Socrate qui provoque le délire dont il affecte d'être lui-même atteint. Le 
${ }^{21}$ Voir notamment à ce sujet un passage du Phèdre, $244 \mathrm{~d} \cdot \mathrm{e}$, qui avait déjà suscité l'in. terrogation particulière d'I. M. Linforth, 1946 : 163.172 dans un article qui faisait suite à son étude du corybantisme, cf. le compte-rendu de Festugière, 1946. II ne nous semble pas que la dimension anthro. pologique du passage ait été correctement évalué. paradoxe se dénoue quand on identifie derrière les logoi de Socrate la figure du dieu auquel lui-même est attaché et qui déclencha l'enthousiasme transmis ensuite d'un maillon à l'autre d'une chaîne possessionnelle (voir aussi la chaîne aimantée de l'Ion, qui transmet de la Muse à l'auditeur l'enthousiasme poétique). Ces logoi comme la présence efficace en Socrate de la mémoire de réalités éternelles, la mémoire du Beau en soi.

De tout ce dossier ne conviendrait-il pas de déduire, tout uniment, que l'avènement si généralement célébré de la raison philosophique correspond à un délire possessionnel, et qu'il ne s'agit peut-être nullement d'une simple image?

Partant de la structure fuguée du rite observée par Cartry dans la diachronie des moments de basculement au cours d'une vie individuelle et la diachronie, d'autre part, de sa propre évolution d'ethnographe, nous avons tâché nous aussi de réintroduire dans une forme de diachronie le rite initiatique des Corybantes, tel que Jeanmaire l'associait, autrefois, sous le nom de Courètes, aux initiations africaines des jeunes garçons. Notre diachronie n'est cependant pas celle des moments qui se répondent dans le cours d'une vie individuelle, mais l'écho, dans le vécu individuel et collectif des Athéniens au tournant du quatrième siècle avant notre ère et dans la théorie de Platon, d'un traumatisme historique, qui n'a pas cessé d'étendre jusqu'à nous le renversement opéré et la frénésie possessionnelle tenue alors pour susceptible de traiter le mal par le mal et de rétablir un semblant d'équilibre. La naissance de la philosophie avec Socrate aussi (et pas seulement avec Parménide ou Empédocle) nous a paru avoir quelque chose à voir avec la possession, calquée sur le délire corybantique qui s'était répandu comme une épidémie dans l'Athènes d'après le désastre de Sicile : mais une possession initiatique masquant la perte au lieu de la révéler. Nous sommes en vérité bien plus près de la malencontre d'un génie de brousse, traitée par l'assignation de la victime au culte de l'instance concernée, que de l'initiation adolescente et des pertes qu'elle provoque ${ }^{21}$. Les contemporains de Socrate, 
dans la bouche de Calliclès, se moquaient de son immaturité politique, de sa tendance à ne fréquenter que les petits jeunes gens pour se monter la tête en leur compagnie dans quelque recoin discret de la ville ${ }^{22}$. Réfugié dans l'atemporel, Socrate autant que Platon paraît avoir su esquiver le passage à l'âge adulte et l'aveu d'une perte historique, et c'était là le symptôme du mal de son temps. Peut-être est-ce aujourd'hui seulement qu'une telle chose a pu nous devenir compréhensible : possédés plus que jamais par les logoi, voire le Logos, ou la sacro-sainte raison scientifique, nous avons laissé nous aussi semble-t-il, sans nous en apercevoir, s'étendre autour de nous le réel de la perte qui désormais se globalise et se nomme dévastation, Verwüstung. Ce serait trop beau si nous avions seulement à avouer une froide étreinte et à chanter dans le deuil :

"Il n'y a rien dans l'enclos où nous sommes assis, sauf le cri de joie des femmes".

22 Platon, Gorgias, $485 b \cdot 486 c$. 


\section{Références bibliographiques}

Blakely, S.

2000, "Madness in the Body Politic: Kouretes, Korybantes, and the Politics of Shamanism ", in J. Hubert (ed.), Madness, Disability and Social Exclusion. The Archaeology and anthropology of Difference, New York : Routledge, pp. 119. 127.

Borgeaud, $\mathrm{P}$

1996 La Mère des dieux : de Cybèle à la Vierge Marie, Paris, Seuil.

Blomart, A.

2000-01 "Identité culturelle, altérité et religions étrangères : exemples antiques de Mithra, Bendis et la Mère des dieux ", Itaca 16-17, pp. 9-22.

Cartry, M.

1987 Sous le masque de l'animal. Essais sur le sacrifice en Afrique noire, Paris, PUF, (Bibliothèque de l'Ecole des Hautes Etudes, Sciences religieuses, 88).

2006 «D'un rite à l'autre : la mémoire du rituel et les remémorations de l'ethnologue ", "Le déni de réalité ", Incidence 2.

2009 "De la divination au sacrifice : la métaphore de l'attache ", in M. Cartry, J.-L. Durand, R. Koch-Piettre, Architecturer l'invisible, Turnhout, Brepols, (Bibliothèque de l'Ecole des Hautes Etudes, Sciences religieuses, 138), p. 307-359.

Detienne, M.

2007 "L'Orphée de la mer Noire ", in Les Dieux d'Orphée, Paris, Gallimard, chap. 1, [1989].

Dodds, E. R.

1977 Les Grecs et l'irrationnel [1959], trad. fr. M. Gibson, Paris, Flammarion, coll. "Champs ", pp. 84-86.

Festugière, A.-J.

1946 "Une nouvelle théorie du sacrifice chez les Grecs ", Revue des Études Grecques, 59, pp. 447-455.

Izard, M. \& Smith, P.

1979 La Fonction symbolique. Essais d'anthropologie, Paris, Gallimard.

Jeanmaire, $\mathrm{H}$.

1939 Couroi et Courètes. Essai sur l'éducation spartiate et sur les rites d'adolescence dans l'Antiquité hellénique, Lille, Bibliothèque universitaire, (Travaux et mémoires de l'Université de Lille, N. S. I, 21).

Lévi-Strauss, C.

1971 L'Homme nu, Paris, Plon, pp. 603. 
Linforth, I. M.

1946 "Telestic madness in Plato, Phaedrus 244D-E. ", University of California Publication in Classical Philology XIII, , 6, pp. 163-172, cf. le compte-rendu de Festugière.

Miller, S. G.

1995 "Old Metroon and Old Bouleuterion in the Classical Agora of Athens ", in M. H. Hansen \& K. Raaflaub (ed.), Studies in the Ancient Greek Polis, Stuttgart, Steiner, pp. 131-156.

Shear, T. L. Jr

1995 "Bouleuterion, Metroon, and the Archives at Athens ", in M. H. Hansen

\& K. Raaflaub (ed.), Studies in the Ancient Greek Polis, Stuttgart : Steiner, pp. 157-190.

Sickinger, J. P.

2004 "The laws of Athens : publication, preservation, consultation ", in E. M. Harris \& L. Rubinstein. The Law and the courts in ancient Greece, Londres, Duckworth, pp. 93-109.

Thompson, H. A. \& Wycherley, R. E.

1972 The Athenian Agora, XIV, Princeton, American School of Classical Studies at Athens, pp. 25-38.

Ustinova, Y.

1992-98 "Corybantism : the Nature and Role of an ecstatic Cult in the Greek Polis ", HOROS, 10-12, pp. 503-520.

Voutiras, E.

1996 "Un culte domestique des Corybantes ", Kernos [en ligne], 9, pp. 243-256. 